\title{
Recursive Identification for Wiener Model With Discontinuous Piece-Wise Linear Function
}

\author{
Han-Fu Chen, Fellow, IEEE
}

\begin{abstract}
This paper deals with identification of Wiener systems with nonlinearity being a discontinuous piece-wise linear function. Recursive estimation algorithms are proposed to estimate six unknown parameters contained in the nonlinearity and all unknown coefficients of the linear subsystem by using the iid Gaussian inputs. The estimates are proved to converge to the corresponding true values with probability one. A numerical example is given to justify the obtained theoretical results.
\end{abstract}

Index Terms-Identification, kernel function, strong consistency, Wiener system.

\section{INTRODUCTION}

$\mathbf{T}$ HE class of dynamic systems consisting of linear subsystems cascaded with memoryless nonlinear blocks appears very important for modeling many practical phenomena in engineering, biology, communication, etc. The system with nonlinearity prior to the linear subsystem is called the Hammerstein system, while it is called the Wiener system if the linear subsystem is followed by a nonlinearity.

For recent years there is an increasing research interest in identification of Hammerstein and Wiener systems, e.g., [1]-[3], [5], [6], [11]-[13], [16], [17], and [19] for Hammerstein systems and [4], [9], [10], [18], and [20] for Wiener systems. For characterizing nonlinearity the previously cited papers are classified into two classes: One uses the parametric approach [1], [3], [4], [6], [9], [17]-[20], and the other one uses the nonparametric approach [2], [5], [10]-[13], [16].

In the parametric approach the nonlinear static block may be presented as a linear combination of known smooth functions, e.g., polynomials; it may also be presented as a piecewise-linear function, which is not necessarily be discontinuous. In such a representation, identification of the nonlinear block is reduced to estimating unknown parameters. In the nonparametric approach there is no structure information about the nonlinearity, and the nonlinear function may be treated by taking its series expansion. In this case identification is equivalent to estimating unknown coefficients in the series expansion, although the number of coefficients in general is infinite. However, the possibly existing discontinuity in nonlinearity may make the series expansion method unsatisfactory. The other nonparametric method is

\footnotetext{
Manuscript received August 26, 2004; revised May 30, 2005. Recommended by Associate Editor E. Bai. This work was supported by the National Natural Science Foundation of China under Grants 60221301, 60334040, and 60474004.

The author is with the Institute of Systems Science, Academy of Mathematics and Systems Science, Chinese Academy of Sciences, Beijing 100080, P. R. China.

Digital Object Identifier 10.1109/TAC.2005.864183
}

directly to estimate the values of the nonlinear function at assigned arguments by using kernel functions.

It is noticed that in most existing results whenever convergence analysis is concerned, only convergence in probability rather than convergence with probability one is established. References [5], [6] may be among a few exceptions.

For identifying Wiener systems a nonparametric approach using kernel functions is presented in [10], where for the linear subsystem the strongly consistent estimates are given for its impulse responses multiplied by an unknown constant $\alpha$, and for the nonlinear part the estimate given in [10] is proven to converge in probability to the product of an unknown constant $\beta$ and the argument corresponding to a pre-assigned value of the nonlinear function. Here the problem is that the nonlinearity in the system must be invertible and its inverse rather than the function itself is estimated, and the unknown parameters $\alpha$ and $\beta$ remain to be defined. In spite of these limitations, the conditions imposed on the system are quite general, in particular, the linear part may be any stable ARMA system.

It is pointed out in [1], [18], [19] that the nonlinearity presented by a piece-wise linear function with preload and dead zone is of importance in applications. It is clear that such kind of nonlinear functions may be discontinuous and cannot be estimated by the method proposed in [10]. Identification of Hammerstein systems with such a nonlinearity is treated in [1], [6], [19], while for Wiener systems it is dealt with in [18]. In this case identification is reduced to estimating unknown parameters contained in both linear and nonlinear blocks.

This paper deals with identification of Wiener systems with nonlinearity being a discontinuous piece-wise linear function. Recursive estimation algorithms are proposed to estimate all unknown coefficients in the system by using the iid Gaussian inputs. The estimates are proved to converge to the corresponding true values with probability one.

The rest of the paper is organized as follows. In Section II the problem is precisely formulated. The coefficients of the nonlinearity are partly estimated by using output data in Section III. Estimating the nonlinear function is completed in Section IV based on the input-output data by using kernel functions. In fact, the variance of the output of the linear subsystem is consistently estimated in this section, but the proof of consistency is placed in the Appendix. The coefficients of the linear subsystem are estimated by the least-squares method [7], [14] in Section V, and the strong consistency of the estimates is proved there as well. A numerical example is demonstrated in Section VI, and a few concluding remarks are given in Section VII. 


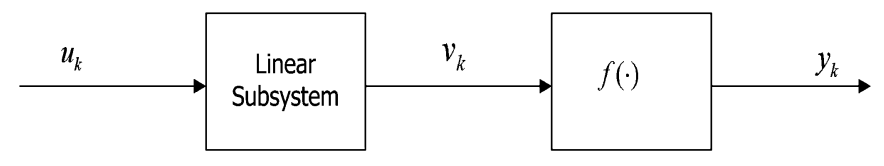

Fig. 1. Wiener system.

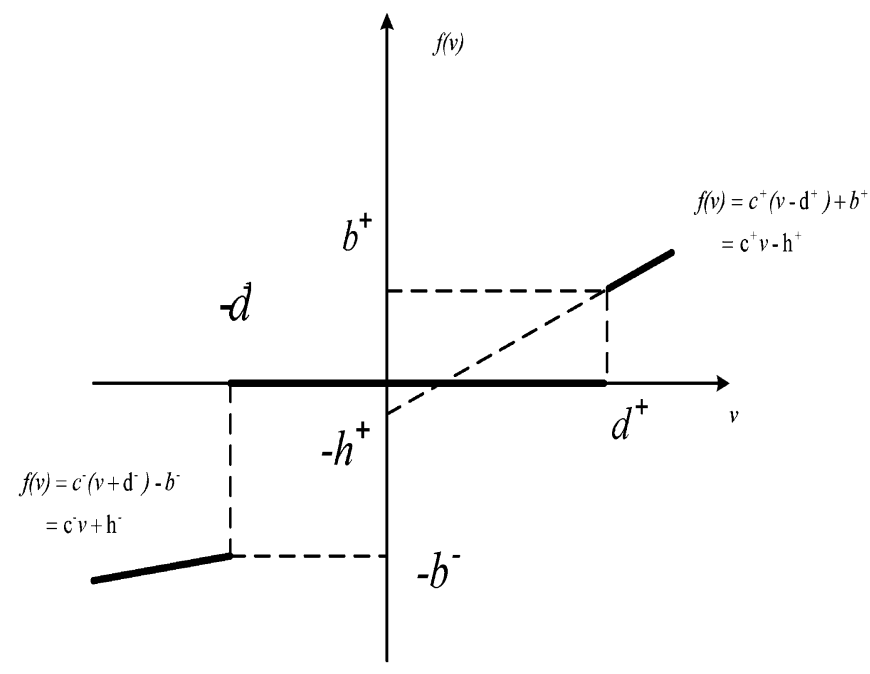

Fig. 2. Nonlinearity.

\section{THE PROBLEM}

Consider the Wiener system expressed by the block diagram shown in Fig. 1.

The nonlinarity of the system is characterized by a static piece-wise linear function

$$
f(v)= \begin{cases}c^{+}\left(v-d^{+}\right)+b^{+}, & v>d^{+} \\ 0, & -d^{-} \leq v \leq d^{+} \\ c^{-}\left(v+d^{-}\right)-b^{-}, & v<-d^{-}, c^{+} \geq 0, c^{-} \geq 0\end{cases}
$$

which is shown in Fig. 2.

Let the system input $u_{k}$ and output $y_{k}$ be connected by

$$
y_{k}=f\left(v_{k}\right) \quad v_{k}=C(z) u_{k}
$$

where

$$
C(z)=1+c_{1} z+\cdots+c_{q} z^{q} .
$$

Denoting by

$$
\theta^{T}=\left[c_{1}, \ldots, c_{q}\right] \text { and } \phi_{k-1}^{T}=\left[u_{k-1}, \ldots, u_{k-q}\right]
$$

the unknown coefficients in the linear subsystem and the regressor, respectively, we rewrite the linear subsystem $v_{k}=C(z) u_{k}$ as

$$
v_{k}=u_{k}+\theta^{T} \phi_{k-1} .
$$

The problem is to design input signal $\left\{u_{k}\right\}$ and on the basis of the input-output data $\left\{u_{k}, y_{k}\right\}$ to recursively estimate all unknown coefficients in both nonlinear and linear parts of the system. Moreover, we want the estimates strongly consistent, i.e., converging to the true values.

The coefficients to be estimated are $c^{+}, d^{+}, b^{+}, c^{-}, d^{-}, b^{-}$ for the nonlinear block, and $\theta$ for the linear subsystem.
As to be seen we will take a sequence of iid random variables to serve as $\left\{u_{k}\right\}$, so $v_{k}$ is stationary. Let $\sigma_{v}^{2}$ denote the variance of $v_{k}$.

For simplicity of writing, let us denote

$$
\alpha^{+}=\frac{d^{+}}{\sigma_{v}} \quad \alpha^{-}=\frac{d^{-}}{\sigma_{v}} \quad \beta^{+}=c^{+} \sigma_{v} \quad \beta^{-}=c^{-} \sigma_{v} .
$$

The parameters $\alpha^{+}, \beta^{+}$, and $h^{+}\left(=-b^{+}+c^{+} d^{+}\right)$are first estimated, and at the same time the estimate for $b^{+}\left(=\alpha^{+} \beta^{+}\right.$ $\left.h^{+}\right)$is derived. Estimation for $\alpha^{-}, \beta^{-}, h^{-}$, and $b^{-}$are carried out in a similar way.

With this done the nonlinearity has not been completely defined yet, since $\sigma_{v}$ is unknown. So, the next step is to estimate $\sigma_{v}$, and the final step is to estimate the coefficient $\theta$ in the linear subsystem.

\section{ESTIMATION FOR $\alpha^{+}, \alpha^{-}, \beta^{+}, \beta^{-}, h^{+}$, AND $h^{-}$}

Let us take a sequence of iid Gaussian random variables $u_{k} \in$ $\mathcal{N}(0,1)$ to serve as the system input. Then the output of the linear subsystem $v_{k}=u_{k}+\theta^{T} \phi_{k-1}$ is Gaussian stationary and ergodic [15]. It is clear that

$$
\sigma_{v}^{2}=1+\|\theta\|^{2} .
$$

Recursively, define

$$
\begin{aligned}
& p_{k}^{+}=\left(1-\frac{1}{k}\right) p_{k-1}^{+}+\frac{1}{k} I_{\left[y_{k}>0\right]} \\
& p_{k}^{-}=\left(1-\frac{1}{k}\right) p_{k-1}^{-}+\frac{1}{k} I_{\left[y_{k}<0\right]}
\end{aligned}
$$

with arbitrary initial values $p_{0}^{+}, p_{0}^{-}$, and then estimate $\alpha^{+}$and $\alpha^{-}$according to the table of $\Phi(x) \triangleq(1 / \sqrt{2 \pi}) \int_{-\infty}^{x} e^{-\left(t^{2} / 2\right)} d t$

$$
p_{k}^{+}=1-\Phi\left(\alpha_{k}^{+}\right) \quad p_{k}^{-}=\Phi\left(-\alpha_{k}^{-}\right) .
$$

Lemma 1: For the system described by (1)-(3), if $\left\{u_{k}\right\}$ is iid with $u_{k} \in \mathcal{N}(0,1)$, then

$$
\alpha_{k}^{+} \underset{k \rightarrow \infty}{\longrightarrow} \alpha^{+} \quad \alpha_{k}^{-} \underset{k \rightarrow \infty}{\longrightarrow} \alpha^{-} \text {a.s. }
$$

where $\alpha_{k}^{+}$and $\alpha_{k}^{-}$are defined by (9).

Proof: By stationarity and ergodicity of $\left\{v_{k}\right\},\left\{y_{k}\right\}$ is also stationary and ergodic, and

$$
p_{k}^{+}=\frac{1}{k} \sum_{i=1}^{k} I_{\left[y_{i}>0\right]} \underset{k \rightarrow \infty}{\longrightarrow} E I_{\left[y_{1}>0\right]} \text { a.s. }
$$

Notice that $c^{+} \geq 0$ and hence " $y_{1}>0$ " is equivalent to " $v_{1}>d^{+}$". Consequently

$$
\begin{aligned}
E I_{\left[y_{1}>0\right]} & =P\left(y_{1}>0\right)=P\left(v_{1}>d^{+}\right) \\
& =\frac{1}{\sqrt{2 \pi}} \int_{\frac{d^{+}}{\sigma v}}^{\infty} e^{-\frac{t^{2}}{2}} d t=1-\Phi\left(\alpha^{+}\right) .
\end{aligned}
$$

Since $\Phi(x)$ is a continuous and increasing function, from (11), (12) it follows that $\alpha_{k}^{+} \longrightarrow k \rightarrow \infty \alpha^{+}$a.s.

The proof for $\alpha_{k}^{-}$is completely similar. 
For estimating $\beta^{+}, h^{+}, \beta^{-}$, and $h^{-}$we recursively calculate

$$
\begin{aligned}
\bar{y}_{k}^{+} & =\left(1-\frac{1}{k}\right) \bar{y}_{k-1}^{+}+\frac{1}{k} y_{k} I_{\left[y_{k}>0\right]} \\
\bar{y}_{k}^{2+} & =\left(1-\frac{1}{k}\right) \bar{y}_{k-1}^{2+}+\frac{1}{k} y_{k}^{2} I_{\left[y_{k}>0\right]} \\
\bar{y}_{k}^{-} & =\left(1-\frac{1}{k}\right) \bar{y}_{k-1}^{-}+\frac{1}{k} y_{k} I_{\left[y_{k}<0\right]} \\
\bar{y}_{k}^{2-} & =\left(1-\frac{1}{k}\right) \bar{y}_{k-1}^{2-}+\frac{1}{k} y_{k}^{2} I_{\left[y_{k}<0\right]}
\end{aligned}
$$

with arbitrary initial values, and obtain estimates $\beta_{k}^{+}$and $h_{k}^{+}$, by solving the following second-order algebraic equations:

$$
\begin{aligned}
\bar{y}_{k}^{+}= & \frac{\beta_{k}^{+}}{\sqrt{2 \pi}} e^{-\frac{\left(\alpha_{k}^{+}\right)^{2}}{2}}-h_{k}^{+} p_{k}^{+} \\
\bar{y}_{k}^{2+}= & \left(\beta_{k}^{+}\right)^{2}\left(\frac{\alpha_{k}^{+} e^{-\frac{-\left(\alpha_{k}^{+}\right)^{2}}{2}}}{\sqrt{2 \pi}}+p_{k}^{+}\right) \\
& -\frac{2}{\sqrt{2 \pi}} \beta_{k}^{+} h_{k}^{+} e^{\frac{-\left(\alpha_{k}^{+}\right)^{2}}{2}}+\left(h_{k}^{+}\right)^{2} p_{k}^{+}
\end{aligned}
$$

where $\alpha_{k}^{+}$, and $p_{k}^{+}$are given by (7) and (9).

Similarly, $\beta_{k}^{-}$and $h_{k}^{-}$are derived from the following algebraic equations:

$$
\begin{aligned}
\bar{y}_{k}^{-}= & -\frac{\beta_{k}^{-}}{\sqrt{2 \pi}} e^{-\frac{\left(\alpha_{k}^{-}\right)^{2}}{2}}+h_{k}^{-} p_{k}^{-} \\
\bar{y}_{k}^{2-}= & \left(\beta_{k}^{-}\right)^{2}\left(\frac{\alpha_{k}^{-} e^{-\frac{\left(\alpha_{k}^{-}\right) 2}{2}}}{\sqrt{2 \pi}}+p_{k}^{-}\right) \\
& -\frac{2}{\sqrt{2 \pi}} \beta_{k}^{-} h_{k}^{-} e^{-\frac{\left(\alpha_{k}^{-}\right)^{2}}{2}}+\left(h_{k}^{-}\right)^{2} p_{k}^{-} .
\end{aligned}
$$

It is worth noting that (17), (18) [or (19), (20)] can easily be solved with respect to $\beta_{k}^{+}$and $h_{k}^{+}$(or $\beta_{k}^{-}$and $h_{k}^{-}$). For this it suffices to replace $h_{k}^{+}$in (18) with

$$
h_{k}^{+}=\frac{1}{p_{k}^{+}}\left(\frac{\beta_{k}^{+}}{\sqrt{2 \pi}} e^{-\frac{\left(\alpha_{k}^{+}\right)^{2}}{2}}-\bar{y}_{k}^{+}\right)
$$

derived from (17). As a result, (18) becomes a second-order algebraic equation with unknown $\beta_{k}^{+}$and its solution is

$$
\beta_{k}^{+}=\left(\frac{\bar{y}_{k}^{2+}-\left(\bar{y}_{k}^{+}\right)^{2} \frac{1}{p_{k}^{+}}}{\alpha_{k}^{+} \gamma_{k}^{+}+p_{k}^{+}-\frac{\left(\gamma_{k}^{+}\right)^{2}}{p_{k}^{+}}}\right)^{1 / 2}
$$

where $\gamma_{k}^{+} \triangleq(1 / \sqrt{2 \pi}) e^{\left.-\left(\left(\alpha_{k}^{+}\right)^{2}\right) / 2\right)}$.

Similarly, we have

$$
h_{k}^{-}=\frac{1}{p_{k}^{-}}\left(\beta_{k}^{-} \gamma_{k}^{-}+\bar{y}_{k}^{-}\right)
$$

and

$$
\beta_{k}^{-}=\left(\frac{\bar{y}_{k}^{2-}-\frac{1}{p_{k}^{-}}\left(\bar{y}_{k}^{-}\right)^{2}}{\alpha_{k}^{-} \gamma_{k}^{-}+p_{k}^{-}-\frac{\left(\gamma_{k}^{-}\right)^{2}}{p_{k}^{-}}}\right)^{1 / 2}
$$

where $\gamma_{k}^{-} \triangleq(1 / \sqrt{2 \pi}) e^{\left.-\left(\left(\alpha_{k}^{-}\right)^{2}\right) / 2\right)}$.
Lemma 2: Under the conditions of Lemma $1, h_{k}^{+}, \beta_{k}^{+}, h_{k}^{-}$, and $\beta_{k}^{-}$given by (13)-(24) are strongly consistent

$$
\begin{aligned}
& h_{k}^{+} \underset{k \rightarrow \infty}{\longrightarrow} h^{+} \quad \beta_{k}^{+} \underset{k \rightarrow \infty}{\longrightarrow} \beta^{+} \\
& h_{k}^{-} \underset{k \rightarrow \infty}{\longrightarrow} h^{-} \quad \beta_{k}^{-} \underset{k \rightarrow \infty}{\longrightarrow} \beta^{-} \text {a.s. }
\end{aligned}
$$

Proof: By ergodicity of $\left\{y_{k}\right\}$, we have

$$
\bar{y}_{k}^{+}=\frac{1}{k} \sum_{i=1}^{k} y_{i} I_{\left[y_{i}>0\right]} \underset{k \rightarrow \infty}{\longrightarrow} E y_{k} I_{\left[y_{k}>0\right]} \text { a.s. }
$$

and

$$
\bar{y}_{k}^{2+}=\frac{1}{k} \sum_{i=1}^{k} y_{i}^{2} I_{\left[y_{i}>0\right]} \underset{k \rightarrow \infty}{\longrightarrow} E y_{k}^{2} I_{\left[y_{k}>0\right]} \text { a.s. }
$$

On the other hand, we see

$$
\begin{aligned}
& E y_{k} I_{\left[y_{k}>0\right]} \\
& \quad=\frac{1}{\sqrt{2 \pi}} \int_{\alpha^{+}}^{\infty} e^{-\frac{t^{2}}{2}}\left(\beta^{+} t-h^{+}\right) d t \\
& =\frac{\beta^{+}}{\sqrt{2 \pi}} e^{-\frac{(\alpha+)^{2}}{2}}-h^{+}\left(1-\Phi\left(\alpha^{+}\right)\right)
\end{aligned}
$$

and

$$
\begin{aligned}
E y_{k}^{2} I_{\left[y_{k}>0\right]} & \frac{1}{\sqrt{2 \pi}} \int_{\alpha^{+}}^{\infty}\left(\beta^{+} t-h^{+}\right)^{2} e^{-\frac{t^{2}}{2}} d t \\
= & \frac{e^{-\frac{(\alpha+)^{2}}{2}} \beta^{+}}{\sqrt{2 \pi}}\left(\alpha^{+} \beta^{+}-2 h^{+}\right) \\
& +\left[\left(\beta^{+}\right)^{2}+\left(h^{+}\right)^{2}\right]\left(1-\Phi\left(\alpha^{+}\right)\right) \\
= & \left(\beta^{+}\right)^{2}\left(\frac{\alpha^{+} e^{-\frac{\left(\alpha^{+}\right)^{2}}{2}}}{\sqrt{2 \pi}}+\left(1-\Phi\left(\alpha^{+}\right)\right)\right) \\
& -\frac{2}{\sqrt{2 \pi}} \beta^{+} h^{+} e^{-\frac{\left(\alpha^{+}\right)^{2}}{2}}+\left(h^{+}\right)^{2}\left(1-\Phi\left(\alpha^{+}\right)\right) .
\end{aligned}
$$

From (27), (28), it follows that

$$
\left(\beta^{+}\right)^{2}=\frac{E y_{k}^{2} I_{\left[y_{k}>0\right]}-\frac{1}{1-\Phi\left(\alpha^{+}\right)} \cdot\left(E y_{k} I_{\left[y_{k}>0\right]}\right)^{2}}{\alpha^{+} \gamma^{+}+\left(1-\Phi\left(\alpha^{+}\right)\right)-\frac{\left(\gamma^{+}\right)^{2}}{1-\Phi\left(\alpha^{+}\right)}}
$$

where

$$
\gamma^{+}=\frac{1}{\sqrt{2 \pi}} e^{-\frac{(\alpha+)^{2}}{2}} .
$$

Noticing $\beta^{+} \geq 0$, by Lemma 1, (25), and (26) we conclude that $\beta_{k}^{+} \longrightarrow k \rightarrow \infty \beta^{+}$a.s. and $h_{k}^{+} \longrightarrow k \rightarrow \infty h^{+}$a.s.

Similar to (28) we have

$E y_{k}^{2} I_{\left[y_{k}<0\right]}$

$$
\begin{aligned}
= & \frac{1}{\sqrt{2 \pi}} \int_{-\infty}^{-\alpha^{-}}\left(\beta^{-} t+h^{-}\right)^{2} e^{-\frac{t^{2}}{2}} d t \\
= & \frac{e^{-\frac{\left(\alpha^{-}\right)^{2}}{2}} \beta^{-}}{\sqrt{2 \pi}}\left(\alpha^{-} \beta^{-}-2 h^{-}\right)+\left[\left(\beta^{-}\right)^{2}+\left(h^{-}\right)^{2}\right] \Phi\left(-\alpha^{-}\right) \\
= & \left(\beta^{-}\right)^{2}\left(\frac{\alpha^{-} e^{-\frac{\left(\alpha^{-}\right)^{2}}{2}}}{\sqrt{2 \pi}}+\Phi\left(-\alpha^{-}\right)\right)-\frac{2}{\sqrt{2 \pi}} \beta^{-} h^{-} e^{-\frac{\left(\alpha^{-}\right)^{2}}{2}} \\
& \left.+\left(h^{-}\right)^{2} \Phi\left(-\alpha^{-}\right)\right)
\end{aligned}
$$


and in a similar way we can prove $\beta_{k}^{-} \longrightarrow k \rightarrow \infty \beta^{-}$a.s. and $h_{k}^{-} \longrightarrow k \rightarrow \infty h^{-}$a.s.

Setting

$$
b_{k}^{+} \triangleq \alpha_{k}^{+} \beta_{k}^{+}-h_{k}^{+} \quad b_{k}^{-}=\alpha_{k}^{-} \beta_{k}^{-}-h_{k}^{-}
$$

by Lemmas 1 and 2 we have

$$
b_{k}^{+} \underset{k \rightarrow \infty}{\longrightarrow} b^{+} \text {a.s., } \quad b_{k}^{-} \underset{k \rightarrow \infty}{\longrightarrow} b^{-} \text {a.s. }
$$

\section{ESTIMATION FOR $c^{+}, d^{+}$AND $c^{-}, d^{-}$}

We have consistently estimated $\alpha^{+}, \beta^{+}, h^{+}$, and $b^{+}$(and $\alpha^{-}, \beta^{-}, h^{-}$, and $\left.b^{-}\right)$. In order to complete estimating $f(\cdot)$ we need to consistently estimate $c^{+}, d^{+}$(and $c^{-}, d^{-}$).

For this, we apply the kernel function approach used in [11], and [12], and also in [5].

Define the kernel function

$$
w_{k} \triangleq k^{-2 \epsilon} e^{-k^{4 \epsilon} u_{k}^{2}} \quad \epsilon \in\left(0, \frac{1}{4}\right)
$$

where $u_{k} \in \mathcal{N}(0,1)$ is the system input defined in Section III.

It is clear that

$$
\begin{aligned}
& E w_{k} \\
& =\frac{k^{2 \epsilon}}{\sqrt{2 \pi}} \int_{-\infty}^{\infty} e^{-k^{4 \epsilon} x^{2}} e^{-\frac{x^{2}}{2}} d x \\
& =\frac{1}{\sqrt{2} \sqrt{2 \pi}} \int_{-\infty}^{\infty} e^{-\frac{y^{2}}{2}} e^{-\frac{k^{-}-4 \epsilon y^{2}}{4}} d y \underset{k \rightarrow \infty}{\longrightarrow} \frac{1}{\sqrt{2}} \\
& E w_{k} y_{k} I_{\left[y_{k}>0\right]} \\
& =E w_{k}\left(c^{+} v_{k}-h^{+}\right) I_{\left[v_{k}>d^{+}\right]} \\
& =k^{2 \epsilon} E e^{-k^{4 \epsilon} u_{k}^{2}}\left(c^{+} u_{k}^{+}+c^{+} \theta^{T} \phi_{k-1}\right. \\
& \left.\quad-h^{+}\right) I_{\left[u_{k}>d^{+}-\theta^{T} \phi_{k-1}\right]} .
\end{aligned}
$$

Noticing $\theta^{T} \phi_{k-1} \in \mathcal{N}\left(0,\|\theta\|^{2}\right)$ and $u_{k}$ is independent of $\theta^{T} \phi_{k-1}$, we then have

$$
\begin{aligned}
E w_{k} y_{k} I_{\left[y_{k}>0\right]} & \frac{1}{2 \pi\|\theta\|} \int_{-\infty}^{\infty} e^{-\frac{y^{2}}{2\|\theta\|^{2}}} \int_{d^{+}-y}^{\infty} e^{-\frac{x^{2}}{2}} e^{-k^{4 \epsilon} x^{2}} \\
& \cdot\left(c^{+} x+c^{+} y-h^{+}\right) d x d y \\
= & \frac{1}{2 \pi\|\theta\|}\left(\int_{-\infty}^{d^{+}}+\int_{d^{+}}^{\infty}\right) e^{-\frac{y^{2}}{2\|\theta\|^{2}}} \int_{\sqrt{2} k^{2 \epsilon}\left(d^{+}-y\right)}^{\infty} \frac{1}{\sqrt{2}} \\
& \cdot e^{-\frac{s^{2}}{2}} e^{-\frac{1}{2}\left(\frac{k^{-2 \epsilon}}{\sqrt{2}}\right)^{2}}\left[\frac{c^{+}}{\sqrt{2}} k^{-2 \epsilon} s+c^{+} y-h^{+}\right] d s d y \\
\underset{k \rightarrow \infty}{\mathrm{a.s.}} & \frac{1}{2 \pi\|\theta\| \sqrt{2}} \int_{d^{+}}^{\infty} e^{-\frac{y^{2}}{2\|\theta\|^{2}}} \int_{-\infty}^{\infty} e^{-\frac{s^{2}}{2}} \\
= & \cdot \frac{1}{\sqrt{2} \sqrt{2 \pi}} \int_{\frac{d^{+}}{\|\theta\|}}^{\infty} e^{-\frac{t^{2}}{2}}\left[-h^{+}+c^{+}\|\theta\| t\right] d t \\
= & \frac{-h^{+}}{\sqrt{2}}\left(1-\Phi\left(\frac{d^{+}}{\|\theta\|}\right)\right)+\frac{c^{+}\|\theta\|}{2 \sqrt{\pi}} \\
& \times e^{-\frac{1}{2}\left(\frac{d^{+}}{\|\theta\|}\right)^{2}} \triangleq \mu .
\end{aligned}
$$

The expectations in (11), (25), and (26) are time-invariant and are approximated by using stationarity and ergodicity of $\left\{y_{k}\right\}$. Although $\left\{w_{k}\right\}$ is no longer stationary, from (33) we see that $E w_{k} y_{k} I_{\left[y_{k}>0\right]}$ converges to a constant $\mu$. The following lemma asserts that the time average of $w_{k} y_{k} I_{\left[y_{k}>0\right]}$ converges to the same constant.

Lemma 3: Under the conditions of Lemma 1

$$
\lim _{n \rightarrow \infty} \frac{1}{n} \sum_{k=1}^{n} w_{k} y_{k} I_{\left[y_{k}>0\right]}=\mu \quad \text { a.s. }
$$

where $\mu$ is given by (33).

The proof is given in the Appendix.

By (5), $c^{+}=\left(\beta^{+} / \sigma_{v}\right), d^{+}=\alpha^{+} \sigma_{v}, c^{-}=\left(\beta^{-} / \sigma_{v}\right), d^{-}=$ $\alpha^{-} \sigma_{v}$. Since we have obtained consistent estimates for $\alpha^{+}, \alpha^{-}, \beta^{+}$, and $\beta^{-}$, in order to estimate $c^{+}, d^{+}, c^{-}$, and $d^{-}$ we need only to estimate $\sigma_{v}=\sqrt{1+\|\theta\|^{2}}$.

In other words, we need to consistently estimate $\|\theta\|$.

Let us recursively compute

$$
\mu_{k}=\left(1-\frac{1}{k}\right) \mu_{k-1}+\frac{1}{k} w_{k} y_{k} I_{\left[y_{k}>0\right]}
$$

where $w_{k}$ is given by (32) and $y_{k}$ is the system output, and find the root of the following algebraic equations with respect to $x$ :

$$
\mu_{k}+\frac{h_{k}^{+}}{\sqrt{2}}\left(1-\Phi\left(\alpha_{k}^{+} x\right)\right)-\frac{\beta_{k}^{+}}{2 \sqrt{\pi} x} e^{-\frac{\left(\alpha_{k}^{+}\right)^{2} x^{2}}{2}}=0 .
$$

Let us check existence and uniqueness of the solution to (36). It is clear that the following function:

$$
g_{k}(x) \triangleq \mu_{k}+\frac{h^{+}}{\sqrt{2}}\left(1-\Phi\left(\alpha^{+} x\right)\right)-\frac{\beta^{+}}{2 \sqrt{\pi} x} e^{-\frac{\left(\alpha^{+}\right)^{2} x^{2}}{2}}
$$

is continuous on $(-\infty, 0)$ and $(0, \infty)$, and its derivative is positive

$$
\begin{aligned}
g_{k}^{\prime}(x) & =\frac{1}{2 \sqrt{\pi}} e^{-\frac{(\alpha+x)^{2}}{2}}\left(-\alpha^{+} h^{+}+\frac{\beta^{+}}{x^{2}}+\left(\alpha^{+}\right)^{2} \beta^{+}\right) \\
& =\frac{1}{2 \sqrt{\pi}} e^{-\frac{(\alpha+x)^{2}}{2}}\left(\frac{\beta^{+}}{x^{2}}+\alpha^{+} b^{+}\right)>0 .
\end{aligned}
$$

It is noticed that with $x$ varying from $-\infty$ to 0 and from 0 to $\infty, g_{k}(x)$ increases from $\mu_{k}+\left(h^{+} / \sqrt{2}\right)$ to $\infty$ and from $-\infty$ to $\mu_{k}$, respectively. Therefore, $g_{k}(x)$ has a unique root on $(0, \infty)$, and hence by Lemmas 1 and 2 there is a unique root for (36) on $(0, \infty)$ for all sufficiently large $\mathrm{k}$. Thus, a numerical method like Newton-Raphson method can be applied to find the root.

Denote by $x_{k}$ the solution of (36), and the estimate $\|\theta\|_{k}$ for $\|\theta\|$ is defined by

$$
\|\theta\|_{k}=\frac{1}{\sqrt{x_{k}^{2}-1}} .
$$

Define

$$
\begin{aligned}
& c_{k}^{+} \triangleq \frac{\beta_{k}^{+}}{\left(1+\|\theta\|_{k}^{2}\right)^{1 / 2}} \quad c_{k}^{-} \triangleq \frac{\beta_{k}^{-}}{\left(1+\|\theta\|_{k}^{2}\right)^{1 / 2}} \\
& d_{k}^{+} \triangleq \alpha_{k}^{+}\left(1+\|\theta\|_{k}^{2}\right)^{1 / 2} \quad d_{k}^{-} \triangleq \alpha_{k}^{-}\left(1+\|\theta\|_{k}^{2}\right)^{1 / 2} .
\end{aligned}
$$


Theorem 1: For the Wiener system described by (1)-(3), if the system input $\left\{u_{k}\right\}$ is iid and $u_{k} \in \mathcal{N}(0,1)$, then $\alpha_{k}^{+}, \alpha_{k}^{-}$ given by (7)-(9), $h_{k}^{+}, h_{k}^{-}, \beta_{k}^{+}, \beta_{k}^{-}$given by (13)-(24), and $c_{k}^{+}, c_{k}^{-}, d_{k}^{+}, d_{k}^{-}$given by (35)-(39) are strongly consistent.

Proof: The strong consistency of $\alpha_{k}^{+}, \alpha_{k}^{-}, h_{k}^{+}, h_{k}^{-}, \beta_{k}^{+}$, and $\beta_{k}^{-}$is proved in Lemmas 1 and 2.

By Lemma $3, \mu_{k}$ given by (35) converges to $\mu$ defined by (33). Therefore, $x_{k}$, the solution of (36), converges to $((1+$ $\left.\left.\|\theta\|^{2}\right)^{1 / 2}\right) /(\|\theta\|)$ a.s., and hence $\|\theta\|_{k} \longrightarrow k \rightarrow \infty\|\theta\|$ a.s. In other words, $\left(1+\|\theta\|_{k}\right)^{1 / 2} \longrightarrow_{k \rightarrow \infty} \sigma_{v}$ a.s., and the strong consistency of $c_{k}^{+}, c_{k}^{-}, d_{k}^{+}$and $d_{k}^{-}$follows.

\section{ESTIMATION OF LINEAR SUBSYSTEM}

By Theorem 1, the nonlinearity $f(\cdot)$ has consistently been estimated, it remains to estimate $C(z)$ in the linear subsystem.

Define

$$
\hat{v}_{k} \triangleq \begin{cases}\frac{1}{\bar{c}_{k}^{+}}\left(h_{k}^{+}+y_{k}\right), & \text { if } y_{k}>0 \\ 0, & \text { if } y_{k}=0 \\ \frac{1}{\bar{c}_{k}^{-}}\left(y_{k}-h_{k}^{-}\right), & \text {if } y_{k}<0\end{cases}
$$

where

$$
\bar{c}_{k}^{+}=c_{k}^{+} \vee \frac{1}{k} \quad \bar{c}_{k}^{-}=c_{k}^{-} \vee \frac{1}{k}
$$

are the modifications of $c_{k}^{+}$and $c_{k}^{-}$and have the same limits as those for $c_{k}^{+}$and $c_{k}^{-}$.

Further, define

$$
\bar{\phi}_{k-1} \triangleq \phi_{k-1} I_{\left[y_{k} \neq 0\right]} \quad \text { and } \quad z_{k} \triangleq\left(\hat{v}_{k}-u_{k}\right) I_{\left[y_{k} \neq 0\right]} \text {. }
$$

By Theorem $1, \epsilon_{k} \triangleq \hat{v}_{k}-v_{k} \longrightarrow k \rightarrow \infty 0$, whenever $y_{k} \neq 0$. Thus, by (4)

$$
\begin{aligned}
z_{k} & =\left(\hat{v}_{k}+\theta^{T} \phi_{k-1}-v_{k}\right) I_{\left[y_{k} \neq 0\right]} \\
& =\left(\theta^{T} \phi_{k-1}+\epsilon_{k}\right) I_{\left[y_{k} \neq 0\right]} \\
& =\theta^{T} \bar{\phi}_{k-1}+\epsilon_{k} I_{\left[y_{k} \neq 0\right]} .
\end{aligned}
$$

The unknown $\theta$ is estimated by the least squares algorithm:

$$
\begin{aligned}
\theta_{k+1} & =\theta_{k}+a_{k} P_{k} \bar{\phi}_{k}\left(z_{k+1}-\theta_{k}^{T} \bar{\phi}_{k}\right) \\
P_{k+1} & =P_{k}-a_{k} P_{k} \bar{\phi}_{k} \bar{\phi}_{k}^{T} P_{k} \\
a_{k} & =\left(1+\bar{\phi}_{k}^{T} P_{k} \bar{\phi}_{k}\right)^{-1}
\end{aligned}
$$

with arbitrary $\theta_{0}$ and $P_{0}>0$.

Theorem 2: Under the conditions of Theorem $1, \theta_{k}$ defined by (41)-(43) is strongly consistent

$$
\theta_{k} \underset{k \rightarrow \infty}{\longrightarrow} \theta \text { a.s. }
$$

Proof: It is well known [7], [14] that the algorithm (43), (44) is the recursive expression of

$$
\begin{aligned}
\theta_{k+1} & =P_{k} \sum_{j=1}^{k-1} \bar{\phi}_{j} z_{k+1}+P_{k} P_{0}^{-1} \theta_{0} \\
& =P_{k} \sum_{j=1}^{k-1} \bar{\phi}_{j} \bar{\phi}_{j}^{T} \theta+P_{k} \sum_{j=1}^{k-1} \bar{\phi}_{j} \epsilon_{j+1}+P_{k} P_{0}^{-1} \theta_{0}
\end{aligned}
$$

and

$$
P_{k}=\left(\sum_{j=1}^{k-1} \bar{\phi}_{j} \bar{\phi}_{j}^{T}+P_{0}^{-1}\right)^{-1} .
$$

By ergodicity, we have

$$
\begin{aligned}
\frac{1}{k} P_{k+1}^{-1}=\frac{1}{k}\left(\sum_{j=1}^{k} \phi_{j} \phi_{j}^{T} I_{\left[y_{j+1} \neq 0\right]}+\right. & \left.P_{0}^{-1}\right) \\
\longrightarrow M<\infty \text { a.s. } &
\end{aligned}
$$

where $M=E \phi_{j} \phi_{j}^{T} I_{\left[y_{j+1} \neq 0\right]}$.

We now show that $M$ is positively definite. For this, it suffices to show

$$
\inf _{\|\xi\|=1} E\left\{\left(\xi^{T} \phi_{j}\right)^{2} I_{\left[y_{j+1} \neq 0\right]}\right\}>0 .
$$

Noticing that

$$
\begin{aligned}
& \Phi\left(-d^{-}-y\right)+1-\Phi\left(d^{+}-y\right) \\
& =1-\frac{1}{\sqrt{2 \pi}} \int_{-d^{-}-y}^{d+-y} e^{-\frac{x^{2}}{2}} d x \\
& \geq 1-\frac{1}{\sqrt{2 \pi}} \int_{-d^{-}-\bar{y}}^{d+-\bar{y}} e^{-\frac{x^{2}}{2}} d x \triangleq \lambda>0 \\
& \quad \forall y \in(-\infty, \infty)
\end{aligned}
$$

where $\bar{y}=\left(\left(d^{+}-d^{-}\right) / 2\right)$, we have

$$
\begin{aligned}
E & \left\{\left(\xi^{T} \phi_{j}\right)^{2} I_{\left[y_{j+1} \neq 0\right]}\right\} \\
& =E\left\{\left(\xi^{T} \phi_{j}\right)^{2} I_{\left[v_{j+1}>d^{+}\right] \cup\left[v_{j+1}<-d^{-}\right]}\right\} \\
& =E\left[\left(\xi^{T} \phi_{j}\right)^{2}\left(\Phi\left(-d^{-}-\theta^{T} \phi_{j}\right)+1-\Phi\left(d^{+}-\theta^{T} \phi_{j}\right)\right)\right] \\
& >\lambda E\left(\xi^{T} \phi_{j}\right)^{2}=\lambda
\end{aligned}
$$

and, hence, $M>0$.

This implies

$$
P_{k+1}=\frac{1}{k}\left(\frac{1}{k} \sum_{j=1}^{k} \bar{\phi}_{j} \bar{\phi}_{j}^{T}+P_{0}^{-1}\right)^{-1} \underset{k \rightarrow \infty}{\longrightarrow} 0 \quad \text { a.s. }
$$

Noticing that

$$
\frac{1}{k}\left|\sum_{j=1}^{k} \bar{\phi}_{j} \epsilon_{j+1}\right| \leq\left(\frac{1}{k} \sum_{j=1}^{k}\left\|\phi_{j}\right\|^{2} \cdot \frac{1}{k} \sum_{j=1}^{k}\left|\epsilon_{j+1}\right|^{2}\right)^{1 / 2} \underset{k \rightarrow \infty}{\longrightarrow} 0
$$

by ergodicity of $\phi_{j}$ and $\epsilon_{j} \longrightarrow{ }_{j \rightarrow \infty} 0$ a.s., we have

$$
\begin{aligned}
P_{k+1} \sum_{j=1}^{k} \bar{\phi}_{j} \epsilon_{j+1}=\left(\frac { 1 } { k } \left(\sum_{j=1}^{k} \bar{\phi}_{j} \bar{\phi}_{j}^{T}\right.\right. & \left.\left.+P_{0}^{-1}\right)\right)^{-1} \\
& \times \frac{1}{k} \sum_{j=1}^{k} \bar{\phi}_{j} \epsilon_{j+1} \underset{k \rightarrow \infty}{\longrightarrow} 0 .
\end{aligned}
$$

This together with (45)-(47) proves the assertion of the theorem.

\section{NUMERICAL EXAMPLES}

We now give a numerical example to demonstrate convergence of the proposed identification algorithms. Matlab is used 


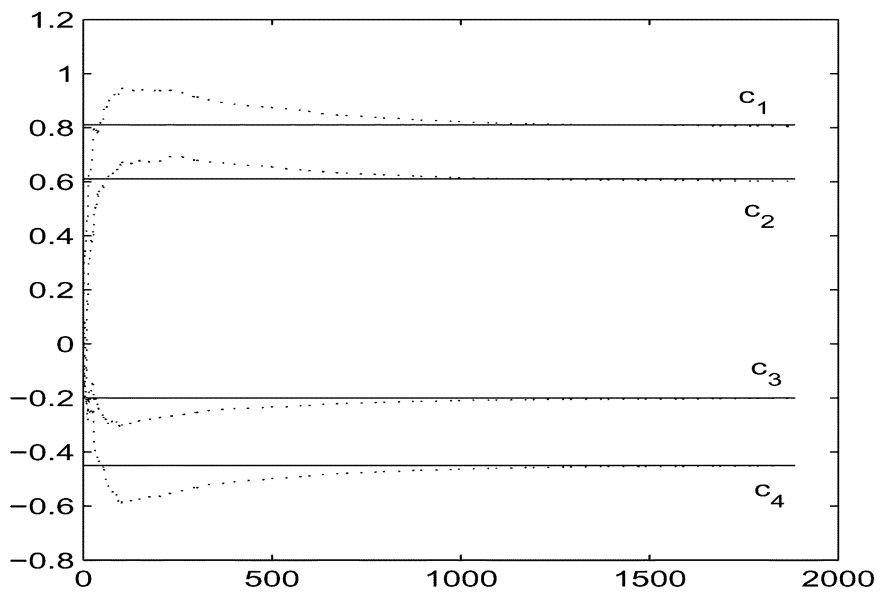

Fig. 3. Estimates for $c_{1}, c_{2}, c_{3}, c_{4}$.

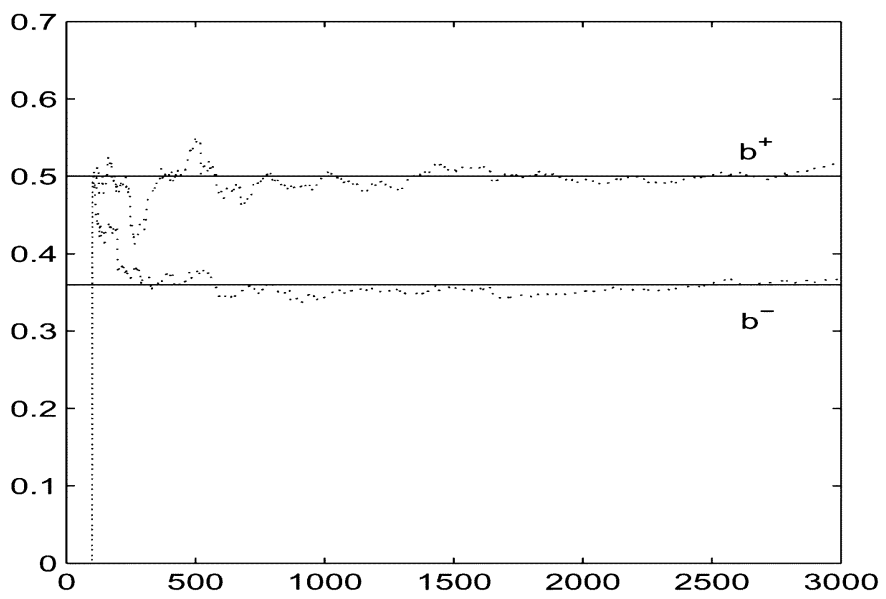

Fig. 4. Estimates for $b^{+}, b^{-}$.

to generate the Gaussian iid random variables $u_{k} \in \mathcal{N}(0,1)$ and to realize all computation.

Let the parameters of $f(v)$ as shown in Fig. 2 be as follows:

$$
\begin{aligned}
& d^{+}=0.60 \quad d^{-}=0.75 \\
& c^{+}=1 \quad c^{-}=0.60 \\
& b^{+}=0.50 \quad b^{-}=0.36
\end{aligned}
$$

and $C(z)$ be of the fourth-order, and let the coefficients in (3) be such that

$$
\begin{aligned}
q & =4 \quad c_{1}=0.81 \quad c_{2}=0.61 \quad c_{3}=-0.2 \quad \text { and } \\
c_{4} & =-0.45 .
\end{aligned}
$$

The parameter $\epsilon$ in the kernel function (32) should be small, because otherwise $w_{k}$ would tend to zero too fast so that the new data would be negligible. Here, we take $\epsilon=(1 / 13500)$.

In order to reduce the influence of the initial values, the parameters $b^{+}, b^{-}, d^{+}$, and $d^{-}$are estimated starting from $k=$ 100 , and the values of their estimates for $k=1, \ldots, 99$ are set to equal zero in the figures.

The simulation results for estimating the unknown parameters are shown in Figs. 3-6, from which we see that all estimates asymptotically tend to the true values. This means that the linear

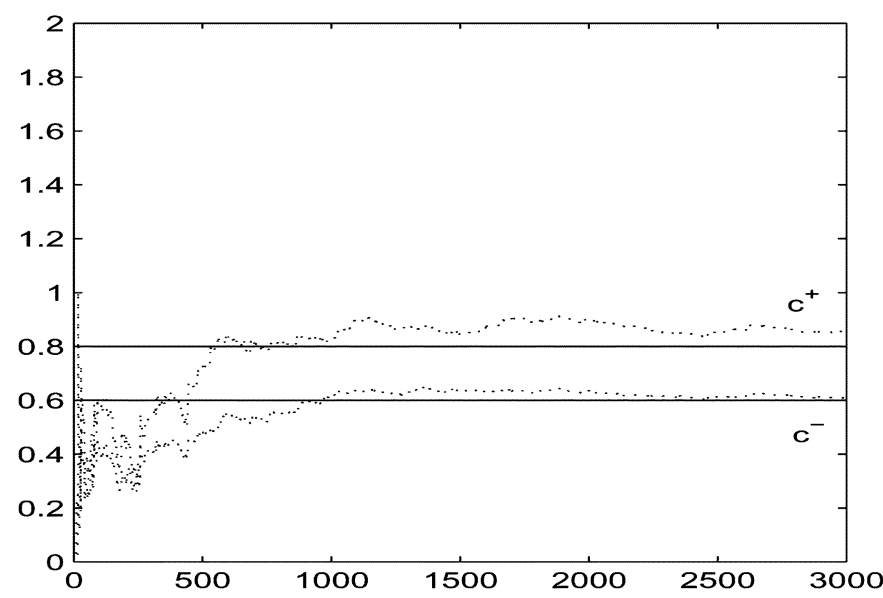

Fig. 5. Estimates for $c^{+}, c^{-}$.

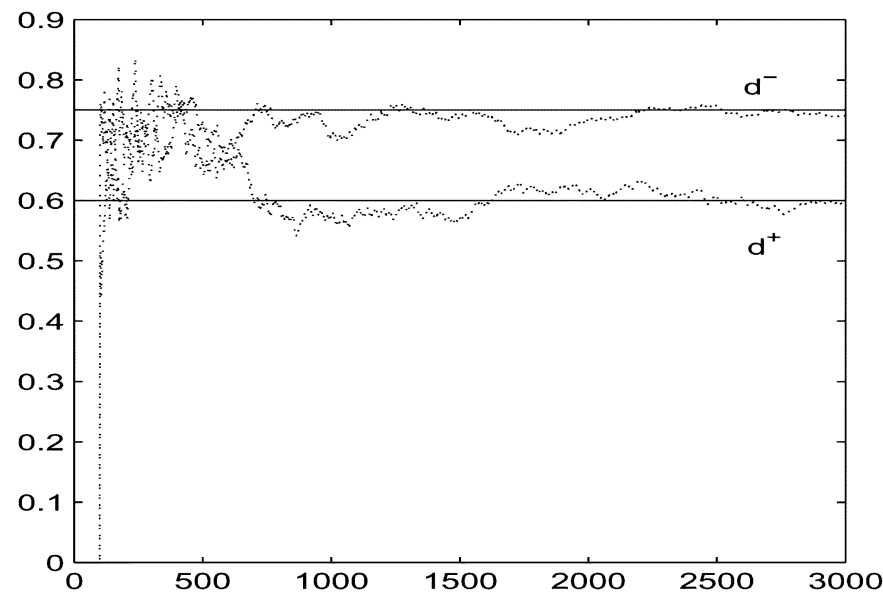

Fig. 6. Estimates for $d^{+}, d^{-}$.

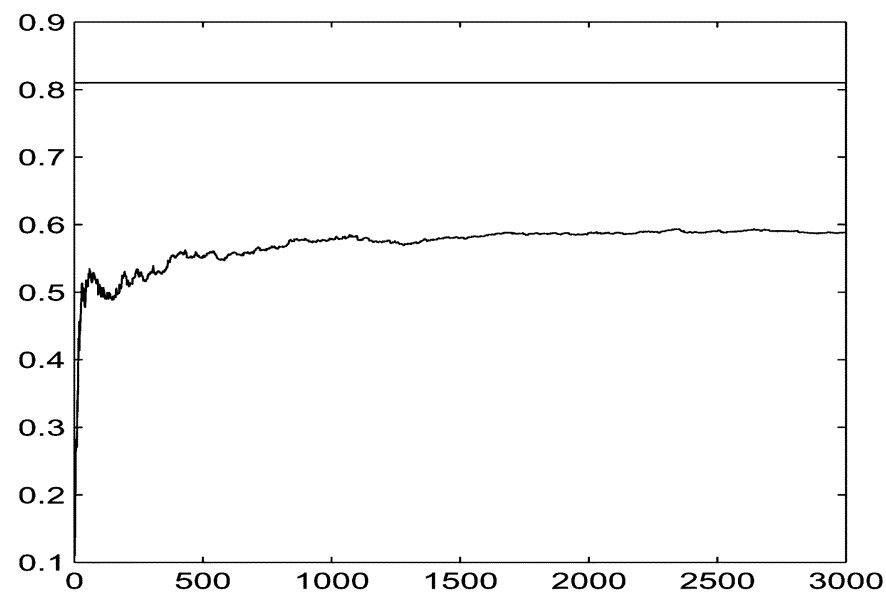

Fig. 7. Estimates for $c_{1}$.

subsystem and the static nonlinearity, i.e., the piecewise linear function, both are well identified.

We now use a naive FIR approach to fit the input-output data into a 4th order MA model ignoring the existence of nonlinearity. Figs. 7-10 show that the estimates for $c_{1}, c_{2}, c_{3}$, and $c_{4}$ 


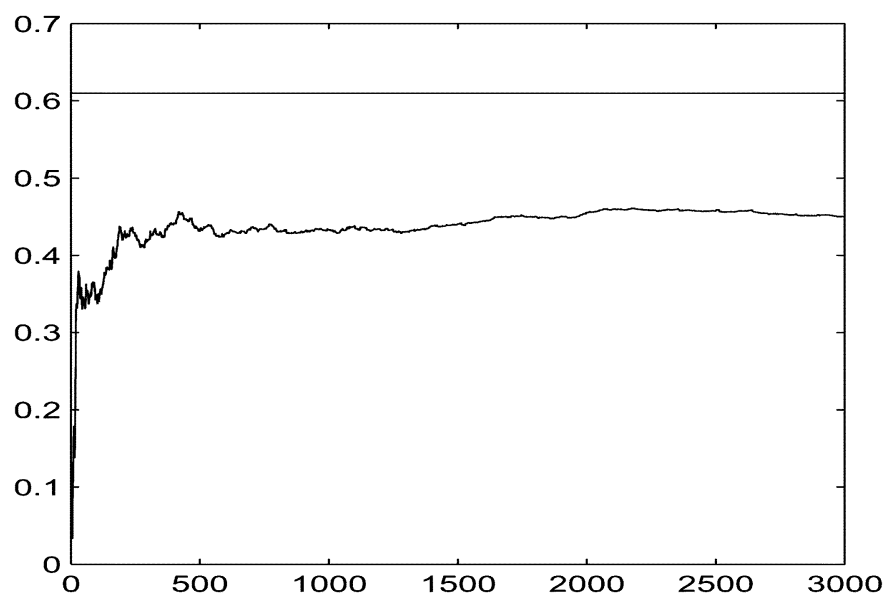

Fig. 8. Estimates for $c_{2}$.

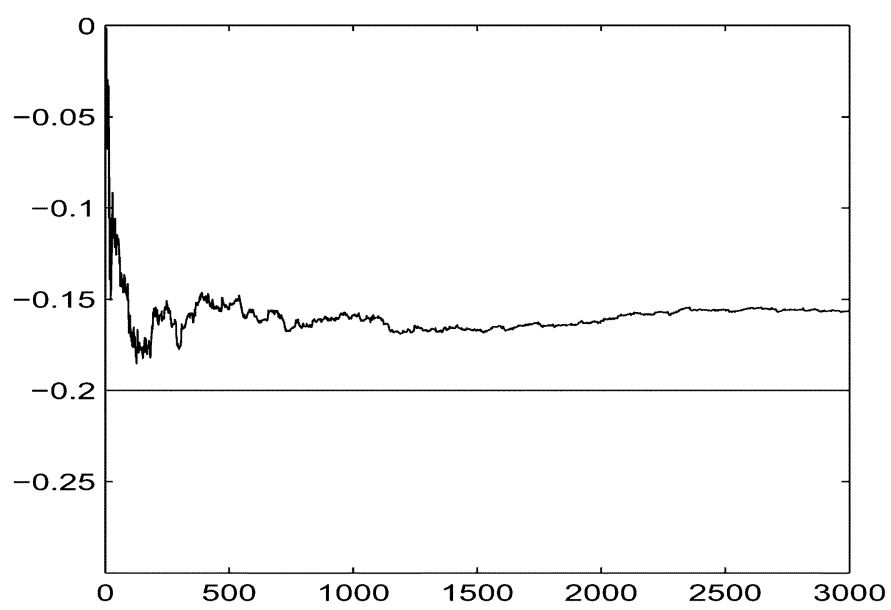

Fig. 9. Estimates for $c_{3}$.

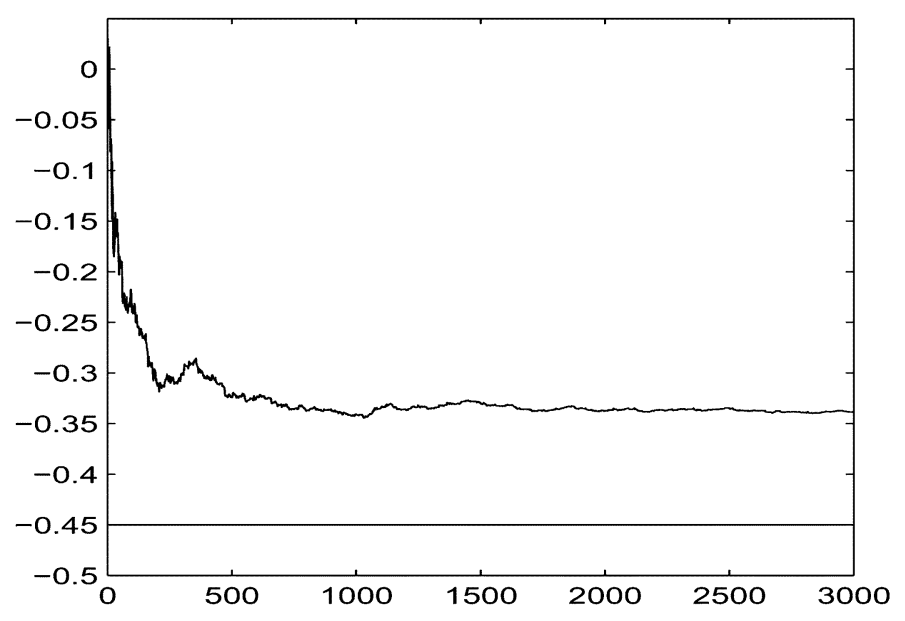

Fig. 10. Estimates for $c_{4}$.

are considerably deviated from the corresponding true values. This means that the static nonlinearity cannot be neglected.

The present paper differs from [18] not only in the estimation methods but also in the models under consideration. The methods given in these two papers, in general, are not comparable. The difference is listed in the following table:

\begin{tabular}{l|c|c}
\hline & Present Paper & Reference [18] \\
\hline Nonlinearity & Six parameters are estimated & $\begin{array}{c}\text { Three parameters are estimated: } \\
d \triangleq d^{+}=d^{-} ; b \triangleq b^{+}=b^{-} ; \\
c \triangleq c^{-}-c^{+}\end{array}$ \\
\hline Linear subsystem & MA & ARMA \\
\hline Estimation method & Recursive & $\begin{array}{c}\text { Nonrecursive: Iteration with } \\
\text { fixed sample size }\end{array}$ \\
\hline Purpose & $\begin{array}{c}\text { To establish strong consistency } \\
\text { for recursive algorithms }\end{array}$ & $\begin{array}{c}\text { To propose algorithms and } \\
\text { illustrative examples }\end{array}$ \\
\hline
\end{tabular}

We take an example computable by both methods given in [18] and in this paper: A fourth-order MA model to serve as the linear subsystem and to restrict the nonlinearity to contain only three parameters. The computational results may not give a fair comparison of these two methods, because they are designed for different models and with different purposes.

\begin{tabular}{l|c|c|c|c|c|c|c}
\hline & $c_{1}$ & $c_{2}$ & $c_{3}$ & $c_{4}$ & $d$ & $b$ & $c^{-}$ \\
\hline True value & 0.81 & 0.51 & -0.2 & -0.45 & 0.3 & 0.5 & 0.6 \\
\hline By [18] & 0.8186 & 0.5104 & -0.1984 & -0.4491 & 0.2751 & 0.4263 & 0.6324 \\
\hline By this paper & 0.7378 & 0.4598 & -0.1861 & -0.4125 & 0.2864 & 0.5074 & 0.6536 \\
\hline
\end{tabular}

The estimates by the method of [18] are computed with available $c^{+}=1$ and with initial values

$$
c_{1}=c_{2}=c_{3}=c_{4}=0 \quad d=0.001 \quad b=0.001 \quad c=0
$$

and the algorithm iterates 8 times with sample size 600 . The estimates by the method given in the paper are at $\mathrm{k}=3000$ with $e=1 / 13000$. For both methods the inputs $u_{k}$ are taken to be iid $\mathcal{N}(0,1)$.

The method of [18], consisting in minimizing estimation errors by iterations, is expected to have a better accuracy than the one given by recursive methods, when the sample size is fixed. This takes place when estimating the linear part, as can be seen from the table. However, for estimating the nonlinear part the table shows a different picture.

\section{CONCLUDING REMARKS}

This paper gives strongly consistent estimates for all parameters contained in the Wiener system with nonlinearity being a discontinuous piece-wise linear function with the help of iid Gaussian inputs. It is worth noting that except the structural assumptions no restrictive conditions are used for convergence of the estimates. The main effort for proving consistency is devoted to estimating the variance $\sigma_{v}$ of not directly observed output of the linear subsystem based on the system input-output data.

For further research it is of interest to consider more general systems, for example, the multidimensional systems and the general ARMA-type linear subsystems. It is also of interest to consider the general nonlinear static block with output observation noise. However, in this case the method used in the present paper may not be suitable, and the stochastic approximation method applied in [5] may be more promising. 


\section{APPENDIX}

Proof of Lemma 3: Since

$$
\begin{aligned}
w_{k} y_{k} I_{\left[y_{k}>0\right]}= & k^{2 \epsilon} e^{-k^{4 \epsilon} u_{k}^{2}} \\
& \times\left(c^{+} u_{k}+c^{+} \theta^{T} \phi_{k-1}-h^{+}\right) \\
& \cdot I_{\left[u_{k}+\theta^{T} \phi_{k-1}>d^{+}\right]}
\end{aligned}
$$

for (34) it suffices to show

$$
\begin{aligned}
& \frac{1}{n} \sum_{k=1}^{n} k^{2 \epsilon} e^{-k^{4 \epsilon} u_{k}^{2}} u_{k} \\
& \quad \times I_{\left[u_{k}+\theta^{T} \phi_{k-1}>d^{+}\right]}^{\longrightarrow} \underset{n \rightarrow \infty}{\longrightarrow} 0 \quad \text { a.s. } \\
& \frac{1}{n} \sum_{k=1}^{n} k^{2 \epsilon} e^{-k^{4 \epsilon} u_{k}^{2}} \\
& \quad \times I_{\left[u_{k}+\theta^{T} \phi_{k-1}>d^{+}\right]} \\
& \quad \underset{n \rightarrow \infty}{\longrightarrow} \frac{1}{\sqrt{2}}\left(1-\Phi\left(\frac{d^{+}}{\|\theta\|}\right)\right) \quad \text { a.s. }
\end{aligned}
$$

and

$$
\begin{aligned}
& \frac{1}{n} \sum_{k=1}^{n} k^{2 \epsilon} e^{-k^{4 \epsilon} u_{k}^{2}} \theta^{T} \\
& \quad \times \phi_{k-1} I_{\left[u_{k}+\theta^{T} \phi_{k-1}>d^{+}\right]} \\
& \underset{n \rightarrow \infty}{\longrightarrow} \frac{\|\theta\|}{2 \sqrt{\pi}} e^{-\frac{1}{2}\left(\frac{d^{+}}{\|\theta\|}\right)^{2}} \text { a.s. }
\end{aligned}
$$

In what follows by $\mathcal{F}_{k}$, we denote the $\sigma$-algebra generated by $\left\{u_{1} \cdots u_{k}\right\}$.

Noticing that

$$
\begin{aligned}
\sup _{k} E\left[\left(k^{\epsilon} e^{-k^{4 \epsilon} u_{k}^{2}} u_{k}\right)^{2} I_{\left[u_{k}+\theta^{T} \phi_{k-1}>d^{+}\right]} \mid \mathcal{F}_{k-1}\right] \\
\quad=k^{2 \epsilon} \frac{1}{\sqrt{2 \pi}} \int_{d^{+}-\theta^{T} \phi_{k-1}}^{\infty} e^{-2 k^{4 \epsilon} u^{2}} u^{2} e^{-\frac{u^{2}}{2}} d u \\
\quad \leq \frac{1}{2 \sqrt{2 \pi}} \int_{-\infty}^{\infty} e^{-\frac{s^{2}}{2}}\left(\frac{s^{2} k^{-4 \epsilon}}{4}\right) e^{-\frac{s^{2} k^{-4 \epsilon}}{8}} d s<\infty
\end{aligned}
$$

by the convergence theorem for martingale difference sequences [7], [8], we have

$$
\begin{aligned}
\sum_{k=1}^{\infty} \frac{1}{k^{1-\epsilon}}\left[k^{\epsilon} e^{-k^{4 \epsilon} u_{k}^{2}} u_{k} I_{\left[u_{k}+\theta^{T} \phi_{k-1}>d^{+}\right]}\right. \\
\left.-E\left[k^{\epsilon} e^{-k^{4 \epsilon u_{k}^{2}}} u_{k} I_{\left[u_{k}+\theta^{T} \phi_{k-1}>d^{+}\right]} \mid \mathcal{F}_{k-1}\right]\right]<\infty
\end{aligned}
$$

By the Kronecker lemma [7], [8]

$$
\begin{aligned}
& \frac{1}{n} \sum_{k=1}^{n} k^{2 \epsilon}\left[e^{-k^{4 \epsilon} u_{k}^{2}} u_{k} I_{\left[u_{k}+\theta^{T} \phi_{k-1}>d^{+}\right]}\right. \\
& \left.-E\left(e^{-k^{4 \epsilon} u_{k}^{2}} u_{k} I_{\left[u_{k}+\theta^{T} \phi_{k-1}>d^{+}\right]} \mid \mathcal{F}_{k-1}\right)\right] \underset{n \rightarrow \infty}{\longrightarrow} 0 \text { a.s. }
\end{aligned}
$$

Further, by noticing

$$
\begin{aligned}
E k^{2 \epsilon} & \left(e^{-k^{4 \epsilon} u_{k}^{2}} u_{k} I_{\left[u_{k}+\theta^{T} \phi_{k-1}>d^{+}\right]} \mid \mathcal{F}_{k-1}\right) \\
= & \frac{1}{\sqrt{2} \sqrt{2 \pi}} \int_{\sqrt{2} k^{2 \epsilon}\left(d^{+}-\theta^{T} \phi_{k-1}\right)}^{\infty}\left(e^{-\frac{s^{2}}{2}} e^{-\frac{s^{2}}{4 k^{4 \epsilon}}}\right) \\
& \times \frac{s}{k^{2 \epsilon}} d s \underset{k \rightarrow \infty}{\longrightarrow} 0
\end{aligned}
$$

from (52) and (53), we conclude (49).
We now show (50). Since

$$
\begin{aligned}
& \sup _{k} E\left[\left(k^{\epsilon} e^{-k^{4 \epsilon} u_{k}^{2}}\right)^{2} I_{\left[u_{k}+\theta^{T} \phi_{k-1}>d^{+}\right]} \mid \mathcal{F}_{k-1}\right] \\
& \quad \leq \sup _{k} E k^{2 \epsilon} e^{-2 k^{4 \epsilon} u_{k}^{2}} \\
& \quad=\frac{1}{\sqrt{2 \pi}} \int_{-\infty}^{\infty} k^{2 \epsilon} e^{-2 k^{4 \epsilon} u^{2}} e^{-\frac{u^{2}}{2}} d u \\
& \quad=\frac{1}{2 \sqrt{2 \pi}} \int_{-\infty}^{\infty} e^{-\frac{s^{2}}{2}} e^{-\frac{s^{2}}{8 k^{4 \epsilon}}} d s<\infty
\end{aligned}
$$

and $\sum_{k=1}^{\infty}\left(\left(1 / k^{1-\epsilon}\right)\right)^{2}<\infty$, again by the convergence theorem for martingale difference sequences [7], [8] we have

$$
\begin{aligned}
\sum_{k=1}^{\infty} \frac{1}{k^{1-\epsilon}} & {\left[k^{\epsilon} e^{-k^{4 \epsilon} u_{k}^{2}} I_{\left[u_{k}+\theta^{T} \phi_{k-1}>d^{+}\right]}\right.} \\
& \left.-E\left(k^{\epsilon} e^{-k^{4 \epsilon} u_{k}^{2}} I_{\left[u_{k}+\theta^{T} \phi_{k-1}>d^{+}\right]} \mid \mathcal{F}_{k-1}\right)\right]<\infty \text { a.s. }
\end{aligned}
$$

From this and the Kronecker lemma, it follows that for (50), it suffices to prove that

$$
\begin{array}{r}
\frac{1}{n} \sum_{k=1}^{n} k^{2 \epsilon} E\left(e^{-k^{4 \epsilon} u_{k}^{2}} I_{\left[u_{k}+\theta^{T} \phi_{k-1}>d^{+}\right]} \mid \mathcal{F}_{k-1}\right) \\
\underset{n \rightarrow \infty}{\longrightarrow} \frac{1}{\sqrt{2}}\left(1-\Phi\left(\frac{d^{+}}{\|\theta\|}\right)\right) \text { a.s. }
\end{array}
$$

Noticing

$$
\begin{aligned}
\int_{\sqrt{2} k^{2 \epsilon}\left(d^{+}-\theta^{T} \phi_{k-1}\right)}^{\infty} e^{-\frac{s^{2}}{2}} & \left(1-e^{-\frac{k^{-} 4 \epsilon s^{2}}{4}}\right) d s \\
\leq & \leq \int_{-\infty}^{\infty} e^{-\frac{s^{2}}{2}}\left(1-e^{-\frac{k^{-4 \epsilon}}{s^{2}} 4}\right) d s \underset{k \rightarrow \infty}{\longrightarrow} 0
\end{aligned}
$$

we have

$$
\begin{aligned}
\lim _{n \rightarrow \infty} & \frac{1}{n} \sum_{k=1}^{n} k^{2 \epsilon} E\left(e^{-k^{4 \epsilon} u_{k}^{2}} I_{\left[u_{k}+\theta^{T} \phi_{k-1}>d^{+}\right]} \mid \mathcal{F}_{k-1}\right) \\
= & \frac{1}{\sqrt{2} \sqrt{2 \pi}} \lim _{n \rightarrow \infty} \frac{1}{n} \sum_{k=1}^{n} \int_{\sqrt{2} k^{2 \epsilon}\left(d^{+}-\theta^{T} \phi_{k-1}\right)}^{\infty} e^{-\frac{s^{2}}{2}} e^{-\frac{k^{-4 \epsilon} s^{2}}{4}} d s \\
= & \frac{1}{\sqrt{2} \sqrt{2 \pi}} \lim _{n \rightarrow \infty} \frac{1}{n} \sum_{k=1}^{n} \int_{\sqrt{2} k^{2 \epsilon}\left(d^{+}-\theta^{T} \phi_{k-1}\right)}^{\infty} e^{-\frac{s^{2}}{2}} d s \\
= & \frac{1}{\sqrt{2} \sqrt{2 \pi}} \lim _{n \rightarrow \infty} \frac{1}{n} \sum_{k=1}^{n} \int_{\frac{1}{\delta}\left(d^{+}-\theta^{T} \phi_{k-1}\right)}^{\infty} e^{-\frac{s^{2}}{2}} d s \\
& \cdot\left(I_{\left[d^{+}-\theta^{T} \phi_{k-1}>0\right]}+I_{\left[d^{+}-\theta^{T} \phi_{k-1}<0\right]}\right) \\
& \left.+\frac{1}{\sqrt{2} \sqrt{2 \pi}} \lim _{n \rightarrow \infty} \frac{1}{n}-\int_{\frac{1}{\delta}\left(d^{+}-\theta^{T} \phi_{k-1}\right)}^{\infty}\right) \\
& \times \sum_{k=1}^{n}\left(\int_{\sqrt{2} k^{2 \epsilon}\left(d^{+}-\theta^{T} \phi_{k-1}\right)}^{\infty} d s\right.
\end{aligned}
$$

where $\delta>0$, and the $\omega$-set $\left[d^{+}-\theta^{T} \phi_{k-1}=0\right]$ is ignored because $\theta^{T} \phi_{k-1} \in \mathcal{N}\left(0,\|\theta\|^{2}\right)$ and, hence, $P \cup_{k=1}^{\infty}\left[d^{+}-\theta^{T} \phi_{k}=\right.$ $0]=0$. 
By ergodicity of $\phi_{k}$, the first term on the right-hand side of (55) equals

$$
\begin{aligned}
& \frac{1}{\sqrt{2} \sqrt{2 \pi}} E \int_{\frac{1}{\delta}\left(d^{+}-\theta^{T} \phi_{k-1}\right)}^{\infty} e^{-\frac{s^{2}}{2}} d s \\
& \cdot\left(I_{\left[d^{+}-\theta^{T} \phi_{k-1}>0\right]}+I_{\left[d^{+}-\theta^{T} \phi_{k-1}<0\right]}\right) \\
& \quad=\frac{1}{\sqrt{2} 2 \pi\|\theta\|}\left(\int_{-\infty}^{d^{+}} e^{-\frac{x^{2}}{2\|\theta\|^{2}}} \int_{\frac{1}{\delta}\left(d^{+}-x\right)}^{\infty} e^{-\frac{s^{2}}{2}} d s d x\right. \\
& \left.\quad+\int_{d^{+}}^{\infty} e^{-\frac{x^{2}}{2\|\theta\|^{2}}} \int_{\frac{1}{\delta}\left(d^{+}-x\right)}^{\infty} e^{-\frac{s^{2}}{2}} d s d x\right)
\end{aligned}
$$

Let $\gamma \in\left(0, d^{+}\right]$. Then the first term on the right-hand side of (56) can be estimated as follows:

$$
\begin{array}{r}
0 \leq \frac{1}{\sqrt{2} 2 \pi\|\theta\|}\left(\int_{-\infty}^{d^{+}-\gamma} e^{-\frac{x^{2}}{2\|\theta\|^{2}}} \int_{\frac{1}{\delta}\left(d^{+}-x\right)}^{\infty} e^{-\frac{s^{2}}{2}} d s d x\right. \\
\left.+\int_{d^{+}-\gamma}^{d+} e^{-\frac{x^{2}}{2\|\theta\|^{2}}} \int_{\frac{1}{\delta}\left(d^{+}-x\right)}^{\infty} e^{-\frac{s^{2}}{2}} d s d x\right) \rightarrow 0
\end{array}
$$

as $\delta \rightarrow 0$ and then $\gamma \rightarrow 0$.

By a similar treatment, we see that the last term of (56) tends to $(1 / \sqrt{2})\left(1-\Phi\left(\left(d^{+} /\|\theta\|\right)\right)\right)$ as $\delta \rightarrow 0$. This combining with (55), (56) we conclude that for (54) it suffices to show that the second term in (55) tends to zero as $\delta \rightarrow 0$. We estimate this term as follows:

$$
\begin{aligned}
& \limsup _{n \rightarrow \infty} \mid \frac{1}{n} \\
& \times \sum_{k=1}^{n}\left(\int_{\sqrt{2} k^{2 \epsilon}\left(d^{+}-\theta^{T} \phi_{k-1}\right)}^{\infty}-\int_{\frac{1}{\delta}\left(d^{+}-\theta^{T} \phi_{k-1}\right)}^{\infty}\right) \\
& \times e^{-\frac{s^{2}}{2}} d s \mid \\
& =\limsup _{n \rightarrow \infty} \mid \frac{1}{n} \\
& \times \sum_{k=1}^{n}\left(\int_{\frac{1}{\delta}\left(d^{+}-\theta^{T} \phi_{k-1}\right)}^{\sqrt{2} k^{2 \epsilon}\left(d^{+}-\theta^{T} \phi_{k-1}\right)} e^{-\frac{s^{2}}{2}} d s\right. \\
& \times I_{\left[d^{+}-\theta^{T} \phi_{k-1}>0\right]} \\
& \left.+\int_{\sqrt{2} k^{2 \epsilon}\left(d^{+}-\theta^{T} \phi_{k-1}\right)}^{\frac{1}{\delta}\left(d^{+}-\theta^{T} \phi_{k-1}\right)} e^{-\frac{s^{2}}{2}} I_{\left[d^{+}-\theta^{T} \phi_{k-1}<0\right]}\right) \mid \\
& \leq \limsup _{n \rightarrow \infty} \frac{1}{n} \sum_{k=1}^{n}\left(\int_{\frac{1}{\delta}\left(d^{+}-\theta^{T} \phi_{k-1}\right)}^{\infty} e^{-\frac{s^{2}}{2}} d s\right. \\
& \times I_{\left[d^{+}-\theta^{T} \phi_{k-1}>0\right]} \\
& \left.+\int_{-\infty}^{\frac{1}{\delta}\left(d^{+}-\theta^{T} \phi_{k-1}\right)} e^{-\frac{s^{2}}{2}} d s I_{\left[d^{+}-\theta^{T} \phi_{k-1}<0\right]}\right) \\
& =E \int_{\frac{1}{\delta}\left(d^{+}-\theta^{T} \phi_{k-1}\right)}^{\infty} e^{-\frac{s^{2}}{2}} d s I_{\left[d^{+}-\theta^{T} \phi_{k-1}>0\right]} \\
& +E \int_{-\infty}^{\frac{1}{\delta}\left(d^{+}-\theta^{T} \phi_{k-1}\right)} e^{-\frac{s^{2}}{2}} d s I_{\left[d^{+}-\theta^{T} \phi_{k-1}<0\right] \underset{\delta \rightarrow 0}{\longrightarrow} 0} 0
\end{aligned}
$$

where the convergence to zero is proved in a way similar to that done for the first term of (56). Thus, we have shown (50). It remains to prove (51).

$$
\text { Set } s_{0}=0 \text { and }
$$

$$
\begin{aligned}
s_{n} \triangleq \sum_{k=1}^{n}\left[k^{\epsilon} e^{-k^{4 \epsilon}} u_{k}^{2} I_{\left[u_{k}+\theta^{T} \phi_{k-1}>d^{+}\right]}\right. & \\
& \left.-E k^{\epsilon} e^{-k^{4 \epsilon} u_{k}^{2}} I_{\left[u_{k}+\theta^{T} \phi_{k-1}>d^{+}\right]} \mid \mathcal{F}_{k-1}\right] \theta^{T} \phi_{k-1} .
\end{aligned}
$$

Noticing that $\sum_{k=1}^{n}\left|\theta^{T} \phi_{k}\right|^{2}=O(n)$ by ergodicity and that

$$
\begin{gathered}
E\left[k^{2 \epsilon} e^{-2 k^{4 \epsilon} u_{k}^{2}} I_{\left[u_{k}+\theta^{T} \phi_{k-1}>d^{+}\right]} \mid \mathcal{F}_{k-1}\right] \\
=\frac{k^{2 \epsilon}}{\sqrt{2 \pi}} \int_{d^{+}-\theta^{T} \phi_{k-1}}^{\infty} e^{-2 k^{4 \epsilon} u^{2}} e^{-\frac{u^{2}}{2}} d u \\
\leq \frac{1}{2 \sqrt{2 \pi}} \int_{-\infty}^{\infty} e^{-\frac{s^{2}}{2}} e^{-\frac{k^{2 \epsilon} s_{s}^{2}}{8}} d s \rightarrow \frac{1}{2}
\end{gathered}
$$

we apply the estimate for weighted sum of martingale difference sequence [7, Th. 2.8] and find that

$$
s_{n}=O(\sqrt{n} \log n)
$$

By using (58) it follows that

$$
\begin{aligned}
\lim _{n \rightarrow \infty} & \sum_{k=1}^{n} \frac{k^{\epsilon}}{k^{1-\epsilon}}\left[e^{-k^{4 \epsilon} u_{k}^{2}} \theta^{T} \phi_{k-1} I_{\left[u_{k}+\theta^{T} \phi_{k-1}>d^{+}\right]}\right. \\
- & \left.E\left(e^{-k^{4 \epsilon} u_{k}^{2}} I_{\left[u_{k}+\theta^{T} \phi_{k-1}>d^{+}\right]} \mid \mathcal{F}_{k-1}\right) \theta^{T} \phi_{k-1}\right] \\
& =\lim _{n \rightarrow \infty} \sum_{k=1}^{n} \frac{1}{k^{1-\epsilon}}\left(s_{k}-s_{k-1}\right) \\
& =\lim _{n \rightarrow \infty} \frac{s_{n}}{n^{1-\epsilon}}+\sum_{k=1}^{n-1} s_{k}\left(\frac{1}{(k-1)^{1-\epsilon}}-\frac{1}{k^{1-\epsilon}}\right) \\
& =\lim _{n \rightarrow \infty} \sum_{k=1}^{n-1} O(\sqrt{k} \log k) O\left(\frac{1}{k(k-1)^{1-\epsilon}}\right) \\
= & \lim _{n \rightarrow \infty} O\left(\sum_{k=1}^{n-1} \frac{\log k}{k^{1 / 2}(k-1)^{1-\epsilon}}\right)<\infty .
\end{aligned}
$$

Therefore, for (51), it suffices to show

$$
\begin{array}{r}
\frac{1}{n} \sum_{k=1}^{n} k^{2 \epsilon} E\left(e^{-k^{4 \epsilon} u_{k}^{2}} I_{\left[u_{k}+\theta^{T} \phi_{k-1}>d^{+}\right]} \mid \mathcal{F}_{k-1}\right) \theta^{T} \phi_{k-1} \\
\underset{n \rightarrow \infty}{\longrightarrow} \frac{\|\theta\|}{2 \sqrt{\pi}} e^{-\frac{1}{2}\left(\frac{d^{+}}{\|\theta\|}\right)^{2}} \text { a.s. }
\end{array}
$$

For this we first show that

$$
\begin{aligned}
& \frac{1}{n} \sum_{k=1}^{n} k^{2 \epsilon} E\left(e^{-k^{4 \epsilon}} u_{k}^{2} I_{\left[u_{k}+\theta^{T} \phi_{k-1}>d^{+}\right]} \mid \mathcal{F}_{k-1}\right) \\
& \cdot I_{\left[d^{+}-\theta^{T} \phi_{k-1}>0\right]} \theta^{T} \phi_{k-1} \underset{n \rightarrow \infty}{\longrightarrow} 0 \text { a.s. }
\end{aligned}
$$


Since by ergodicity $\lim _{n \rightarrow \infty}(1 / n) \sum_{k=1}^{n}\left|\theta^{T} \phi_{k}\right|^{2}<\infty$ a.s., for (60) we need only to show

$\frac{1}{n} \sum_{k=1}^{n} k^{4 \epsilon}\left[E\left(e^{-k^{4 \epsilon} u_{k}^{2}} I_{\left[u_{k}+\theta^{T} \phi_{k-1}>d^{+}\right]} \mid \mathcal{F}_{k-1}\right)\right.$

$$
\left.\cdot I_{\left[d^{+}-\theta^{T} \phi_{k-1}>0\right]}\right]^{2} \underset{n \rightarrow \infty}{\longrightarrow} 0
$$

which is equivalent to

$$
\begin{array}{r}
\frac{1}{n} \sum_{k=1}^{n}\left|\int_{\sqrt{2} k^{2 \epsilon}\left(d^{+}-\theta^{T} \phi_{k-1}\right)}^{\infty} e^{-\frac{s^{2}}{2}} e^{-\frac{k^{-4 \epsilon_{s}}}{4}} d s\right|^{2} \\
\cdot I_{\left[d^{+}-\theta^{T} \phi_{k-1}>0\right]} \underset{n \rightarrow \infty}{\longrightarrow} 0
\end{array}
$$

for which it suffices to show

$$
\begin{aligned}
& \frac{1}{n} \sum_{k=1}^{n} \mid \int_{\sqrt{2} k^{2 \epsilon}\left(d^{+}-\theta^{T} \phi_{k-1}\right)}^{\infty}\left.e^{-\frac{s^{2}}{2}} d s\right|^{2} \\
& \times I_{\left[d^{+}-\theta^{T} \phi_{k-1}>0\right] \underset{n \rightarrow \infty}{\longrightarrow} 0} 0 \text { a.s. }
\end{aligned}
$$

Proceeding as before, we estimate the left-hand side as follows:

$$
\begin{aligned}
& \lim _{n \rightarrow \infty} \frac{1}{n} \sum_{k=1}^{n}\left|\int_{\sqrt{2} k^{2 \epsilon}\left(d^{+}-\theta^{T} \phi_{k-1}\right)}^{\infty} e^{-\frac{s^{2}}{2}} d s\right|^{2} I_{\left[d^{+}-\theta^{T} \phi_{k-1}>0\right]} \\
& \leq 2 \lim _{n \rightarrow \infty} \frac{1}{n} \sum_{k=1}^{n}\left|\int_{\frac{\left(d^{+}-\theta^{T} \phi_{k-1}\right)}{\delta}}^{\infty} e^{-\frac{s^{2}}{2}} d s\right|^{2} I_{\left[d^{+}-\theta^{T} \phi_{k-1}>0\right]} \\
& \quad+\lim _{n \rightarrow \infty} \frac{2}{n} \sum_{k=1}^{n}\left[\left(\int_{\sqrt{2} k^{2 \epsilon}\left(d^{+}-\theta^{T} \phi_{k-1}\right)}^{\infty}\right) e^{-\frac{s^{2}}{2}} d s\right]^{2} I_{\left[d^{+}-\theta^{T} \phi_{k-1}>0\right]}^{\infty}
\end{aligned}
$$

where on the right-hand side the first term tends to zero as $\delta \rightarrow 0$ as can be shown by the treatment similar to that carried out in (57), while the second term is bounded by

$$
\begin{aligned}
\lim _{n \rightarrow \infty} \frac{2}{n} \sum_{k=1}^{n}\left(\int_{\frac{\left(d^{+}-\theta^{T} \phi_{k-1}\right)}{\delta}}^{\infty} e^{-\frac{s^{2}}{2}} d s\right)^{2} \\
\quad \times I_{\left[d^{+}-\theta^{T} \phi_{k-1}>0\right] \underset{\delta \rightarrow 0}{\longrightarrow} 0 .} .
\end{aligned}
$$

Thus, we have proved (60), and hence for (59), it is sufficient to show

$$
\begin{aligned}
\lim _{n \rightarrow \infty} \frac{1}{n} \sum_{k=1}^{n} k^{2 \epsilon} E & \left(e^{-k^{4 \epsilon} u_{k}^{2}} I_{\left[u_{k}+\theta^{T} \phi_{k-1}>d^{+}\right]} \mid \mathcal{F}_{k-1}\right) \\
\cdot I_{\left[d^{+}-\theta^{T} \phi_{k-1}<0\right]} \theta^{T} \phi_{k-1} & =\frac{\|\theta\|}{2 \sqrt{\pi}} e^{-\frac{1}{2}\left(\frac{d^{+}}{\|\theta\|}\right)^{2}}
\end{aligned}
$$

or to show

$$
\begin{gathered}
\lim _{n \rightarrow \infty} \frac{1}{n} \sum_{k=1}^{n} \frac{1}{2 \sqrt{\pi}} \int_{\sqrt{2} k^{2 \epsilon}\left(d^{+}-\theta^{T} \phi_{k-1}\right)}^{\infty} e^{-\frac{s^{2}}{2}} \\
\times e^{-\frac{k^{-4 \epsilon} s^{2}}{4}} d s \theta^{T} \phi_{k-1} \\
\cdot I_{\left[d^{+}-\theta^{T} \phi_{k-1}<0\right]}=\frac{\|\theta\|}{2 \sqrt{\pi}} e^{-\frac{1}{2}\left(\frac{d^{+}}{\|\theta\|}\right)^{2}}
\end{gathered}
$$

for which in turn it suffices to show

$$
\begin{aligned}
\lim _{n \rightarrow \infty} \frac{1}{n} \sum_{k=1}^{n} \int_{\sqrt{2} k^{2 \epsilon}\left(d^{+}-\theta^{T} \phi_{k-1}\right)}^{\infty} e^{-\frac{s^{2}}{2}} d s \theta^{T} \phi_{k-1} \\
\cdot I_{\left[d^{+}-\theta^{T} \phi_{k-1}<0\right]}=\|\theta\| e^{-\frac{1}{2}\left(\frac{d^{+}}{\|\theta\|}\right)^{2}} .
\end{aligned}
$$

Similar to (63) (64) it is shown that

$$
\begin{aligned}
\lim _{n \rightarrow \infty} \frac{1}{n} \sum_{k=1}^{n}\left[\left(\int_{\sqrt{2} k^{2 \epsilon}\left(d^{+}-\theta^{T} \phi_{k-1}\right)}^{\infty}\right) e^{-\frac{s^{2}}{2}} d s\right]^{2} I_{\left[d^{+}-\theta^{T} \phi_{k-1}<0\right]} \\
\left.-\int_{\frac{\left(d^{+}-\theta^{T} \phi_{k-1}\right)}{\delta}}^{\infty} e^{-\frac{s^{2}}{2}} d s\right)^{2} \\
\leq \lim _{n \rightarrow \infty} \frac{1}{n} \sum_{k=1}^{n}\left(\int_{-\infty}^{\frac{d^{+}-\theta^{T} \phi_{k-1}}{\delta}}\right. \\
\quad \times I_{\left[d^{+}-\theta^{T} \phi_{k-1}<0\right]} \\
=\frac{1}{\sqrt{2 \pi}\|\theta\|} \int_{d^{+}}^{\infty}\left(\int_{-\infty}^{\frac{d^{+}-x}{\delta}} e^{-\frac{s^{2}}{2}} d s\right) \\
\quad \times e^{-\frac{x^{2}}{2\|\theta\|^{2}}} d x \underset{\delta \rightarrow 0}{\longrightarrow} 0 .
\end{aligned}
$$

Consequently

$$
\begin{aligned}
& \lim _{n \rightarrow \infty} \frac{1}{n} \sum_{k=1}^{n} \int_{\sqrt{2} k^{2 \epsilon}\left(d^{+}-\theta^{T} \phi_{k-1}\right)}^{\infty} e^{-\frac{s^{2}}{2}} d s \theta^{T} \phi_{k-1} \\
& \cdot I_{\left[d^{+}-\theta^{T} \phi_{k-1}<0\right]} \\
& \quad=\lim _{\delta \rightarrow 0} \lim _{n \rightarrow \infty} \frac{1}{n} \sum_{k=1}^{n} \int_{\frac{d^{+}-\theta^{T} \phi_{k-1}}{\delta}}^{\infty} e^{-\frac{s^{2}}{2}} d s \\
& \times \theta^{T} \phi_{k-1} I_{\left[d^{+}-\theta^{T} \phi_{k-1}<0\right]}^{\infty} \\
& \quad=\lim _{\delta \rightarrow 0} \frac{1}{\sqrt{2 \pi}\|\theta\|} \int_{d^{+}} e^{-\frac{x^{2}}{2\|\theta\|^{2}}} \\
& \times \int_{\frac{d^{+}-x}{\delta}}^{\infty} e^{-\frac{s^{2}}{2}} d s x d x \\
& \quad=\lim _{\delta \rightarrow 0} \frac{1}{\sqrt{2 \pi}} \int_{\frac{d^{+}}{\|\theta\|}}^{\infty} e^{-\frac{t^{2}}{2}} t\|\theta\| \\
& \times \int_{\frac{d^{+}-t\|\theta\|}{\delta}}^{\infty} e^{-\frac{s^{2}}{2}} d s d t=\|\theta\| e^{-\frac{1}{2}\left(\frac{d^{+}}{\|\theta\|}\right)^{2}} .
\end{aligned}
$$

Thus, we have proved (66), which in turn proves (65) and (59). This means that (51) has been proved and the proof of the lemma is completed.

\section{ACKNOWLEDGMENT}

The author would like to thank Mr. X.-L. Hu for his efficient computer simulation.

\section{REFERENCES}

[1] E. W. Bai, "Identification of linear systems with hard input nonlinearities of known structure," Automatica, vol. 38, no. 5, pp. 853-860, 2002.

[2] - "Frequency domain identification of Hammerstein models," IEEE Trans. Autom. Control, vol. 48, no. 4, pp. 530-542, Apr. 2003. 
[3] H. Al-Duwaish and M. N. Karim, "A new method for the identification of Hammerstein model," Automatica, vol. 33, no. 10, pp. 1871-1875, 1997.

[4] P. Celka, N. J. Bershad, and J. M. Vesin, "Stochastic gradient identification of polynomial Wiener systems: Analysis and application," IEEE Trans. Signal Process., vol. 49, pp. 301-313, 2001.

[5] H. F. Chen, "Path-wise convergence of recursive identification algorithms for Hammerstein systems," IEEE Trans. Autom. Control, vol. 49, no. 10, pp. 1641-1649, Oct. 2004.

[6] — "Strong consistency of recursive identification for Hammerstein systems with discontinuous piecewise-linear memoryless block," IEEE Trans. Autom. Control, 2006, to be published.

[7] H. F. Chen and L. Guo, Identification and Stochastic Adaptive Control. Boston, MA: Birkhäuser, 1991.

[8] Y. S. Chow and H. Teicher, Probability Theory. New York: SpringerVerlag, 1978

[9] A. D. Kalafatis, L. Wang, and W. R. Cluett, "Identification of Wienertype nonlinear systems in a noisy environment," Int. J. Control, vol. 66, pp. 923-941, 1997.

[10] W. Greblicki, "Nonparametric approach to Wiener system identification," IEEE Trans. Circuits Syst. I: Fundam. Theory Appl., vol. 44, no. 6, pp. 538-545, Jun. 1997.

[11] - "Stochastic approximation in nonparametric identification of Hammerstein systems," IEEE Trans. Autom. Control, vol. 47, no. 11, pp. 1800-1810, Nov. 2002.

[12] W. Greblicki and M. Pawlak, "Nonparametric identification of Hammertein systems," IEEE Trans. Inform. Theory, vol. 35, no. 3, pp. 409-418, Mar. 1989.

[13] Z. Q. Lang, "A nonparametric polynomial identification algorithm for the Hammerstein system," IEEE Trans. Autom. Control, vol. 42, no. 10, pp. 1435-1441, Oct. 1997.

[14] L. Ljung, System Identification. Englewood Cliffs, NJ: Prentice-Hall, 1987.

[15] M. Loève, Probability Theory. New York: Springer-Verlag, 1977.
[16] M. Pawlak, "On the series expansion appraoch to the identification of Hammertein system," IEEE Trans. Autom. Control, vol. 36, no. 6, pp. 763-767, Jun. 1991.

[17] P. Stoica and T. Söderstrom, "Instrumental-variable methods for identification of Hammerstein systems," Int. J. Control, vol. 35, no. 3, pp. 459-476, 1982.

[18] J. Vörös, "Parameter identification of Wiener systems with discontinuous nonlinearities," Syst. Control Lett., vol. 44, pp. 363-372, 2001.

[19] _ "Recursive identification of Hammerstein systems with discontinuous nonlinearities containing dead-zeros," IEEE Trans. Autom. Control, vol. 48, no. 12, pp. 2203-2206, Dec. 2003.

[20] T. Wigren, "Convergence analysis of recursive identification algorithms based on the nonlinear Wiener model," IEEE Trans. Autom. Control, vol. 39, no. 12, pp. 2191-2206, Dec. 1994.

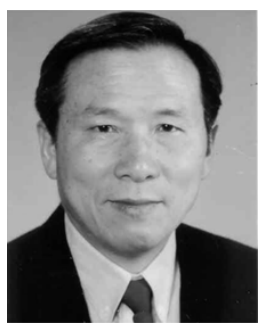

Han-Fu Chen (SM'94-F'97) graduated from Leningrad (St. Petersburg) State University, Russia, in 1961 .

He joined the Institute of Mathematics, Chinese Academy of Sciences (CAS), Beijing, China, in 1961. Since 1979, he has been with the Institute of Systems Science, which now is a part of the Academy of Mathematics and Systems Science, CAS. He is a Professor of the Laboratory of Systems and Control of the Institute. His research interests are mainly in stochastic systems, including system identification, adaptive control, and stochastic approximation and its applications to systems, control, and signal processing. He has authored and coauthored more than 160 journal papers and seven books. 